\title{
Sonozaki syndrome in the spotlight of imaging
}

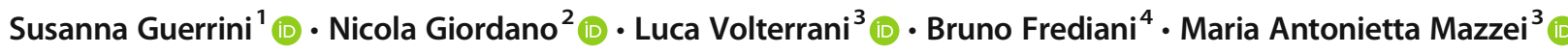

Received: 3 August 2020 / Revised: 30 September 2020 / Accepted: 18 October 2020 / Published online: 22 October 2020

(C) International League of Associations for Rheumatology (ILAR) 2020

\section{Presentation}

Sonozaki syndrome (SS) also known as pustulotic arthroosteitis (PAO) is a rare chronic inflammatory disease belonging to the group of spondyloarthritis $(\mathrm{SpA})$, firstly described by Sonozaki [1]. Onset is frequently seen at age 30-40, with a similar prevalence between sexes. Sterile palmoplantar pustular lesions and anterior thoracic joint involvement (with $77 \%$ of manubrium sterni) are the most typical findings, although other joints can be involved (34\% of axial, $32 \%$ of peripheral joints, and $13 \%$ of sacroiliitis). We present the case of a 30-year-old male with palmoplantar pustulosis (PPP) and painful swelling of the sternum, clavicles, and lower ribs. The patient had no family history of other rheumatic disorders. General physical examination resulted negative for clinical involvement of the spinal column, and of the sacroiliac and peripheral joints, with no signs or symptoms of infective disease. Laboratory findings showed mild increase of erythrocyte sedimentation rate (ESR) $(50 \mathrm{~mm} / \mathrm{h})$ and C-reactive protein (CRP) $(2.5 \mathrm{mg} / \mathrm{dl}$, n.v. 0.5); other biochemical tests (blood count, serum electrolytes, creatinine, urea, uric acid, glucose, bilirubin, transaminases, creatine phosphokinase, LDH, alcaline phosphatase), protein levels with electrophoresis, and general tests of urine, immunoglobulins, and complement and anti-CCP were normal; rheumatoid factor (RA test and Waaler rose) and antinuclear antibodies (ANA) were negative. HLA typing resulted negative for aplotipo HLAB51, 52, and 27. Skin biopsy confirmed the suspected PAO diagnosis. The evaluation of the patient at 6 and 12 months confirmed the complete recovery after therapy with $16 \mathrm{mg}$ of metilprednisolone with descending sequences of dose for 2 months.

\section{Discussion}

Sternocostoclavicular hyperostosis (SSCH) represented by diffuse sclerosis in the sternum, clavicle, or ribs in the advanced phase and by inflammatory enthesopathy of the costoclavicular ligament, small hyperostotic foci at the sternal end of ribs, and isolated periosteal reaction involving the clavicles in the early phase are characteristic radiological findings of SS, usually accompanied by erosive and sclerotic processes of the manubriosternal joint, as expression of arthritis [2] (Fig. 1). Spondylodiscitis in the vertebral involvement and sclerosis/hyperostosis in the sacroiliac joints may be also observed, whereas the peripheral joint involvement of SS is usually not severe and non-erosive. The latter may suggest the differential diagnosis with psoriatic arthrisis (PsA), where the peripheral joint involvement is common and with erosive course, and more generally with $\mathrm{SpA}$ involving the anterior chest wall, in the form of arthritis of the sternoclavicular, manubriosternal, and sternocostal joints, such changes that have sometimes been termed as Tietze's syndrome [3-6].
Susanna Guerrini

guerrinisus@gmail.com

1 Unit of Diagnostic Imaging, Department of Radiological Sciences, Azienda Ospedaliero-Universitaria Senese, "Santa Maria alle Scotte" General Hospital, Viale Mario Bracci, 16, 53100 Siena, Tuscany, Italy
2 Scleroderma Unit, Department of Medical, Surgical and Neuro Sciences, University of Siena, Siena, Italy

3 Unit of Diagnostic Imaging, Department of Medical, Surgical and Neuro Sciences and of Radiological Sciences, University of Siena, Azienda Ospedaliero-Universitaria Senese, Siena, Italy

4 Unit of Rheumatology, Department of Medical, Surgical and Neuro Sciences, University of Siena, Azienda Ospedaliero-Universitaria Senese, Siena, Italy 

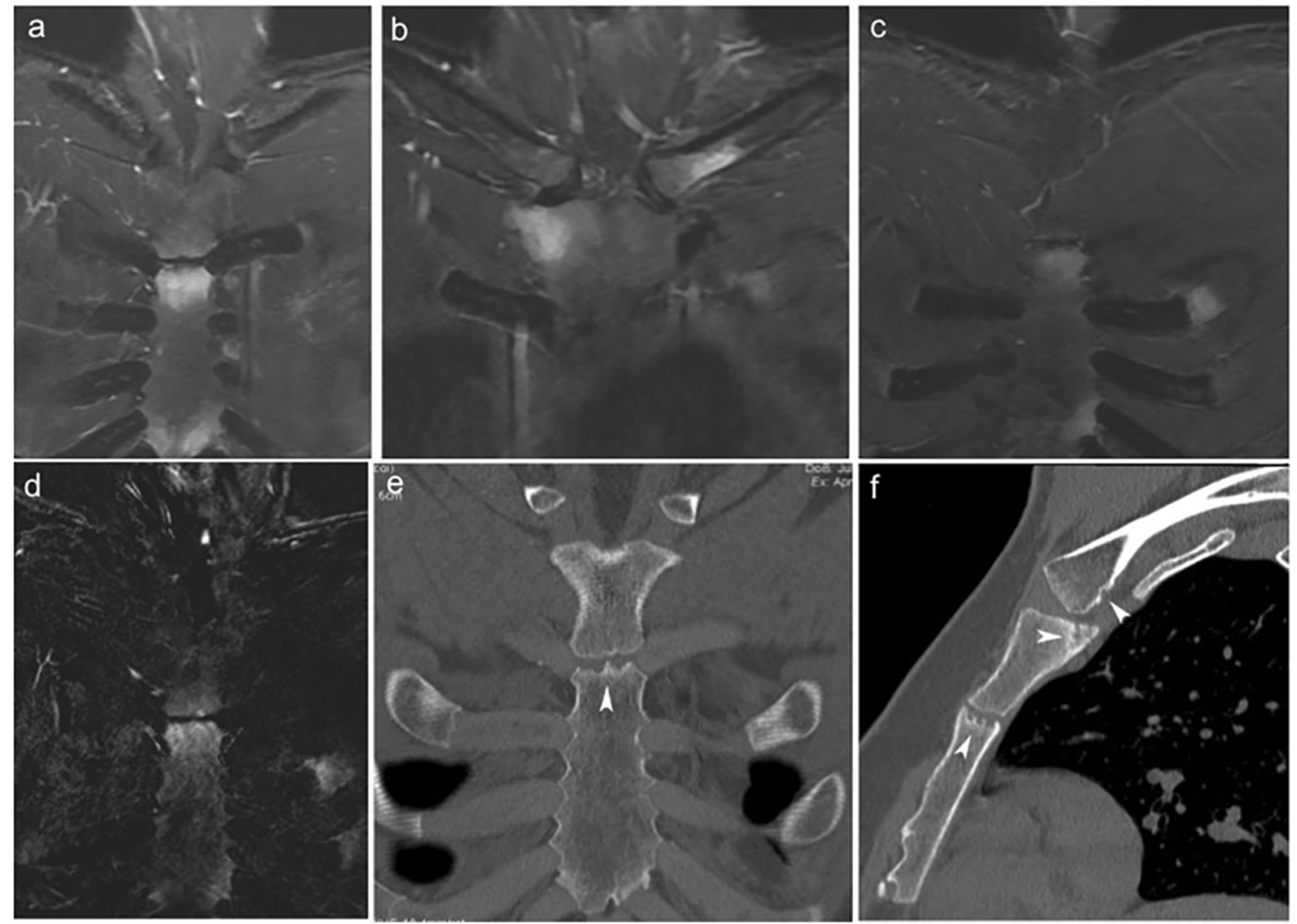

Fig. 1 MRI (a-d) and CT (e, f). MRI examination showed subcondral edema (a-c) and osteitis (enhancement of subcondral bone marrow after contrast media administration on $\mathrm{T} 1$ gradient echo image with fat saturation, d) on the body and manubrium of the sternum, on the periosteal clavicle and on some ribs, as expression of arthritis. CT better

\section{Compliance with ethical standards}

- The manuscript is original work of authors. All data, tables, figures, etc. used in the manuscript are prepared originally by authors; otherwise, the sources are cited and reprint permission is attached.

- The manuscript has not been and will not be published elsewhere or submitted elsewhere for publication.

- The study complies with current ethical considerations.

\section{Disclosures None.}

Patient consent All clinical images have patient consent for publication.

\section{References}

1. Sonozaki H, Mitsui H, Miyanaga Y, Okitsu K, Igarashi M, Hayashi Y, Matsuura M, Azuma A, Okai K, Kawashima M (1981) Clinical features of 53 cases with pustulotic arthro-osteitis. Ann Rheum Dis 40:547-553. https://doi.org/10.1136/ard.40.6.547 demonstrated erosive and subtle sclerotic processes of the MSJ and manubrium and tiny erosions of the inferior aspect of the periosteum of the sternal end of the clavicle (arrow heads). The final diagnosis, supported by both clinical data and imaging features, was Sonozaki syndrome

2. Hyodoh K, Sugimoto H (2001) Pustulotic arthro-osteitis: defining the radiologic spectrum of the disease. Semin Musculoskelet Radiol 5:89-93. https://doi.org/10.1055/s-2001-15658

3. La Paglia E, Zawaideh JP, Lucii G, Mazzei MA (2019) MRI of the axial skeleton: differentiating non-inflammatory diseases and axial spondyloarthritis: a review of current concepts and applications: special issue on "musculoskeletal imaging of the inflammatory and degenerative joints: current status and perspectives". Radiol Med 124: 1151-1166. https://doi.org/10.1007/s11547-019-01045-5 Review

4. Volterrani L, Mazzei MA, Giordano N, Nuti R, Galeazzi M, Fioravanti A (2008) Magnetic resonance imaging in Tietze's syndrome. Clin Exp Rheumatol 26:848-853

5. Guerrini S, Bagnacci G, Barile A, La Paglia E, Gentili F, Luzzi L et al (2020) Anterior chest wall non-traumatic diseases: a road map for the radiologist. Acta Biomed 91(8-S):43-50. https://doi.org/10. 23750/abm.v91i8-S.9972

6. Guglielmi G, Scalzo G, Cascavilla A, Salaffi F, Grassi W (2008) Imaging of the seronegative anterior chest wall (ACW) syndromes. Clin Rheumatol 27:815-821. https://doi.org/10.1007/s10067-0080905-1

Publisher's note Springer Nature remains neutral with regard to jurisdictional claims in published maps and institutional affiliations. 